\title{
"Universal Data Elements," or the Biopolitical Life of Homeless Populations*
}

\author{
Craig Willse ${ }^{1}$
}

\begin{abstract}
This article explores the development of the Homeless Management Information Systems program by the U.S. Department of Housing and Urban Development. Launched in 2001, the program mandates data collection by homeless social service agencies receiving federal funds. The program is examined in terms of broader responses to the "electronic turn" in social work. The generative capacities of database management systems are understood as producing surveillance at a register other than the individual subject often presumed in political theories of social control as well as surveillance studies of information technologies. The article argues that we must move toward an understanding of homeless management as a biopolitical enterprise, rather than only a disciplinary one. A discussion of how the HMIS program produces a homeless population as an object of knowledge and plane of intervention provides an understanding of the significance of HMIS for homelessness in the U.S. as well as for surveillance studies more broadly.
\end{abstract}

\section{Introduction}

This article seeks to explore a number of relationships: between social welfare and social control; between information technologies and surveillance technologies; between neoliberal governance and its objects of rule. It also seeks to understand how each set of relationships implicates the others, leading to questions that include: How do information technologies of surveillance transform, and not merely enable, the capacities of neoliberal governance? What becomes of subject identity positions in a techno-political context of digitization? How are knowledge and intervention mutually constituted through governance and surveillance?

In order to begin exploring these relationships and answering these questions, this article

\footnotetext{
* The research for this article benefited from my participation in the Workshop on Surveillance and Inequality, hosted by Arizona State University, and in a year-long seminar hosted by the Center for Place, Culture and Politics at The Graduate Center, City University of New York. I thank the organizers of both for inviting me to join, and the participants of both for important and helpful conversations. Tracy Chu, Ananya Mukherjea, Rachel Schiff, Neil Smith, three anonymous reviewers and editors Jill Fisher and Torin Monahan offered thoughtful feedback on earlier drafts. I thank Dean Spade, Greg Goldberg and especially Patricia Clough for rigorous and generous engagement and critique with this and all my work.

${ }^{1}$ Program in Sociology, The Graduate Center, City University of New York. mailto:cwillse@gc.cuny.edu
} 
takes on the specific case of database surveillance systems used in the United States to count and assess homeless populations. A directive of the federal government being rolled out in private non-profit agencies that receive funding from the Department of Housing and Urban Development (HUD), the Homeless Management Information Systems (HMIS) program raises important questions about the nature of both surveillance and governance, and suggests that the individual subject presumed by surveillance studies may not be the only or even primary target of surveillance technologies. By situating the turn to computer-based record keeping in a history of social service technologies, the article begins to map out the place of information technologies and social service programs in a post-welfare or neoliberal state. Too much literature in the field of surveillance studies has presumed a monolithic system of surveillance, ignoring the differential impact and uneven application of surveillance technologies. While HUD's HMIS program fits within a broader move to quantitative measures of health and social services, due to the particular political and social vulnerabilities of homeless populations, it also has specific implications for understanding how surveillance systems maintain, exploit, and reproduce forms of inequality.

Making note of HUD's use of the term “Homeless Management Information System” suggests the kinds of political and social implications a study of this program might yield. The term "information management" is of course a common one in fields of information technology, and so the designation "Homeless Information Management System" would appear to be the obvious one. In fact, that designation has been in use prior to HUD's program. However, in HUD's use, the two internal terms (information/management) are flipped, and so rather we have a "Homeless Management Information System." I will hope to show that this slip takes place not only because HUD here is managing the homeless (something different than "serving," as a sociology of medicine and theories of social control would make clear). But I posit that this slip (whether or not it's accidental) follows from an exchangeability of homeless and information that is the result of the technical rendering of them as commensurate through the databasing of homelessness. This flip of terms, the exchange of information for homeless, signals the possibility that the direct management of one may simultaneously be the management of the other. Thus the exchange is not a moment of cognitive dissonance, but a murmur suggesting we think about how trading between information and homeless management becomes conceptually, which is to say technically, possible. Ultimately, I argue that this occurs through the organization of the national homeless population as a population of what HUD calls "universal data elements."

The first part of the article offers a description of the program and how it fits within the broad work of the Department of Housing and Urban Development. The second part considers the technical capacities of HUD's universal data elements in terms of databases and networks. I then move on to consider debates about the uses of computer technologies in social work, as well as some of the limits of these debates. Drawing from surveillance studies, I suggest that we must grapple with the productive capacities of surveillance technologies. This leads to the suggestion that HUD's program functions to produce knowledge and intervention of a homeless population that cannot be reduced to any of its constituent parts. Understanding this requires a reconceptualizing of surveillance systems as biopolitical, rather than only disciplinary, technologies. The study hopes to contribute to a substantive understanding about the management of 
homeless populations in the contemporary United States, and the role of surveillance technologies in these projects. It furthermore hopes to contribute to theoretical formulations in surveillance studies that can account for the production of social stratification, inequality, and vulnerability through surveillance systems.

\section{Managing the Homeless, Managing Information}

For much of its history, the federal government of the United States has remained largely uninvolved in issues of homelessness, preferring to allocate (if only by default) responsibility to state and local branches of government. The short-lived Federal Emergency Relief Administration (FERA), in existence from just 1932-34, marked the only component of the New Deal to specifically address housing and service provision for a homeless population that grew along with economic depression (Snow and Anderson, 1993:14-15). Following FERA's dissolution, the provision of shelter for homeless populations fell back upon an ad-hoc matrix of state and municipal programs and charitable, often church-based, organizations (Rossi, 1989:22-25).

A series of federal agencies charged with housing issues existed from the 1930s on, but did not deal with homelessness. Rather, these agencies (which were re-organized as HUD in 1965-66) dealt first with increasing home-ownership and providing rental assistance for low-income families, and later became involved in "urban renewal" programs of redeveloping low-income neighborhoods, often by tearing down so-called slum housing (Thompson 2006:1-8). Only at the end of its second decade of existence did HUD specifically take on homelessness as part of its broad mission of "housing and urban development." This occurred through the 1987 McKinney-Vento Homeless Assistance Act, which authorized a variety of federal initiatives (such as funding streams for locallyadministered shelter and supportive housing programs) and created an intra-agency board charged with oversight of federal homeless programs. ${ }^{2}$ Through its McKinney-Vento programs, HUD began providing funding to private agencies already in existence and addressing homeless populations, and has subsequently spurred the further growth of such agencies. $^{3}$ With the McKinney-Vento Act, the federal government returns, but not to the same context as the early New Deal of FERA. HUD's role in administering homeless populations today emerges, rather, in relation to a context that writers describe as the end of the welfare state, the withering of civil society, or the launch of neoliberalism (Clough, 2004; Rose, 1999; Hardt, 1995; Deleuze, 1992; Burchell et al, 1991). Within this context, contemporary governance is characterized by a re-configuration of the state, the public sphere, and private industry, such that the ability to distinguish one from the other becomes increasingly difficult (Clough, 2007). At the level of institutions, this reconfiguration has pressured the boundaries that previously delimited the enclosures of the hospital, the prison, the school, the military, the workplace, and the family, as well as the social service agency, and has furthermore helped bleed those boundaries into a public sphere once thought to be outside institutional or state control. Central to this

\footnotetext{
2 The act was originally named the Stewart B. McKinney Act.

3 The growth of a homeless service industry is explored by Funiciello (1994) and Lyon-Callo (2004). For a general discussion of the emergence of a "non-profit industrial complex" of which homeless social services would be a part, see INCITE! Women of Color Against Violence (2007).
} 
reorganization is an incomplete but profound shift from state-based to private management, or a complex and unpredictable mix of state/corporate authority over those institutional domains. From a perspective of the history of (the death of) the U.S. welfare state and the social service provision associated with it, we can note the end of New Deal era programs, a shift to locally administered and private programs, the rise of rhetoric calling for "individual responsibility," a decrease in available government funding, and an increase in barriers to service access (See Mink, 1998).

Whereas FERA was directly administered by the federal government, and included employment in public works projects as well as housing services, HUD's work is comprised of setting policy, providing funding, and monitoring services offered by state, city and private agencies. Thus HUD plays a piloting role, setting parameters and facilitating between state, municipal, and private entities. HUD, and other Departments of the federal government, might therefore be conceptualized most usefully as management centers (as in a corporate model) rather than as traditionally governing bodies. The fact that HUD does not offer employment programs like FERA, but rather provides loans and funds (not only to non-profit agencies, but also to private real-estate developers involved in the social and economic reorganization of U.S. urban centers) encapsulates perfectly the realignment of private and public interests and activities that characterize contemporary neoliberal governance.

Of course, HUD funding comes along with restrictions on the use of funds and requirements about reporting agency activities to HUD. Along these lines, in 2001 Congress mandated that HUD collect national data on the homeless population of the United States as well as data on the effectiveness of federally funded programs targeting this population. The 2001 Congressional directive required local jurisdictions receiving McKinney-Vento funds to produce "unduplicated counts" of homeless populations they served toward an aggregation of this data on a national level. As a Senate report stated:

[ . . . ] HUD must collect data on the extent of homelessness in America as well as the effectiveness of the McKinney homeless assistance programs in addressing this condition. These programs have been in existence for some 15 years and there has never been an overall review or comprehensive analysis on the extent of homelessness or how to address it. The Committee believes that it is essential to develop an unduplicated count of homeless people, and an analysis of their patterns of use of assistance (HUD McKinney homeless assistance as well as other assistance both targeted and not targeted to homeless people), including how they enter and exit the homeless assistance system and the effectiveness of assistance (Office of the Federal Register, 2003:43431).

Efforts to meet this directive have subsequently become embedded in the requirements attached to McKinney-Vento funds, particularly through an initiative to foster the use among service agencies of an HMIS database system. Since 2004, HUD has required all agencies receiving McKinney-Vento funds to implement the use of an HMIS, and it has developed a number of technical support programs to facilitate this. 
HMIS designates any information management software application, of which many versions exist, that a service agency uses for recording "client-level information on the characteristics and service needs of homeless persons ... A An HMIS is typically a webbased software application that homeless assistance providers use to coordinate service provision, manage their operations, and better serve their clients" (U.S Department of Housing and Urban Development, 2006b:1). Promoting HMIS among agencies it funds has assisted HUD with the two tasks named in the Senate Report cited above-producing a national count of homelessness and assessing patterns of use in programs targeting homelessness.

Though HUD's initiative is fairly recent, the use of computer-based applications for managing client records is not new in homeless service provision. As with other sectors of social and medical care, the move from paper to databank has been in motion for some time now, a shift some have designated the "electronic turn" (Garrett, 2005:531). Within a history of social work practices, the use of HMIS has been understood as replacing a "case notes" system of an earlier era of service provision, in which a social worker produced narrative accounts of clients in terms of their needs, services accessed, and changes in their condition and circumstance. An HMIS is not, however, only a computerbased repository of notes (which might imply, for example, typing up case notes in a word-processing program). Rather, it is a database management system that provides fixed inquiry fields (demographic details, categories of need, kinds of service) in which standardized measures are input. The use of an HMIS does not signal simply a shift from the pen stroke to the keystroke, but from qualitative to quantitative records and assessments. Consequently, an HMIS changes not just how case notes are recorded, but what can be recorded, and in what forms.

Agencies participating in HUD's HMIS program may choose to develop their own version of an HMIS or, more likely, purchase an already existing, customizable HMIS program from any number of private vendors. ${ }^{4}$ This is important to note. HUD itself does not provide an HMIS application, or require that agencies use any specific version of an HMIS. What it does require, and what marks its entry into HMIS, is the collection of what it calls "universal data elements" in forms that meet data standards set by HUD. Simply put, universal data elements are a set of data that each agency must collect on each client it serves. These elements include data such as name, social security number, and gender as well as data related to service, including program entry and exit dates. ${ }^{5}$ Providing data standards, or requirements as to the form of this data, HUD ensures that these elements will be commensurate across agencies. For example, data standards outlined for the universal data element "Name of client" are as follows:

\footnotetext{
4 HUD provides links to over thirty vendors that sell McKinney-Vento compliant HMIS software packages. See http://hmis.info/Defaut.aspx?classicAsp=products_info.asp. Accessed 18 December 2007.

5 The universal data elements are Name, Social Security Number, Date of Birth, Ethnicity/Race, Gender, Veteran's Status, Disabling Condition, Residence Prior to Program Entry, Zip Code of Last Permanent Address, Program Entry/Exit Date. HUD additionally requires what it calls "program-specific data elements” for specific streams of McKinney-Vento funding, such as its Housing Opportunities for People with AIDS (HOPWA) program (U.S. Department of Housing and Urban Development, 2006).
} 
Data Source: Client interview or administered form.

When Data Are Collected: Upon initial program entry or as soon as possible thereafter.

Subjects: All clients.

Definition and Instructions: Four fields should be created in the HMIS database to capture the client's full first, middle, and last names and any suffixes (e.g., John David Doe, Jr.). Try to obtain legal names only and avoid aliases or nicknames. Section 5 of this Notice discusses how to treat missing information for open-ended questions.

Required Response Categories: Current name: First name, middle name, last name, suffix; Other Name Used to Receive Services previously: First name, middle name, last name suffix (Office of the Federal Register, 2003:43439).

HUD argues that requiring the use of universal data elements in standard form will correct miscounts of homeless populations accessing services at multiple locations. Collected from dispersed locales by agencies using any number of software applications, these data will then provide the raw material for the aggregate population counts and trends analysis required by Congress, as data produced at each locale will be commensurate in form and thus can be set to calculation together. As HUD states:

The universal data standards will make possible unduplicated estimates of the number of homeless people accessing services from homeless providers, basic demographic characteristics of people who are homeless, and their patterns of service use. The universal data standards will also allow measurement of the number and share of chronically homeless people who use homeless services. The standards will enable generation of information on shelter stays and homelessness episodes over time (Office of the Federal Register, 2004:45890).

Along these lines, using data collected through HMIS programs funded by HUD, in February 2007 HUD presented the "Annual Homeless Assessment Report to Congress," drawing from a sample of 80 local communities participating in HUD's HMIS program (U.S. Department of Housing and Urban Development, 2007a).

Of course, what HUD considers an information technology, others would consider a technology of surveillance. ${ }^{6}$ Early critics of HUD's program pointed in Orwellian terms to the "Big Brother" feel of the program, accusing the government of spying upon and tracking a vulnerable population. HUD's response to these accusations emphasizes that its program does not create a national database of homeless people. Databases are

\footnotetext{
6 There is a discussion to be had, which is beyond the scope of this article, about whether any information technology is at least potentially, if not in practice, a technology of surveillance. In other words, does the process of rendering informatic automatically open up to surveillance?
} 
maintained at local levels by Continuums of Care, formalized networks of agencies providing homeless services within a community (See Wong et al, 2006 and Lyon-Callo, 2004:11-23) that may determine what information is shared amongst agencies. HUD, in turn, only collects data at the aggregate level, not at the level of the individual case. The names and social security numbers of clients, for example, remain at the local level.

While this attends to some of the privacy concerns of clients, advocates and critics of surveillance technologies alike, we should nonetheless ask what, if not a national database, HUD is in fact creating. I argue that its formation of "the universal data element” is not only HUD's unique contribution to HMIS, but its contribution to surveillance systems and governance more broadly. I hope to show that the universal data element acts as a connector between two technological forms central to governance today - the database and the network - and thereby allows data to be simultaneously local and national, public and anonymous, databased and networked. As such, the universal data element is significant not as a discrete object, but as a force for arranging technical relationships between technologies, agencies, clients, and HUD. I will furthermore argue that if we understand HUD's HMIS program in terms of surveillance technologies, it is a form of surveillance not only or even primarily interested in the individual homeless person. If, following one move in surveillance studies, we understand surveillance technologies as constitutive of the very objects they track, we must ask what is constituted, produced or organized by HUD's HMIS program. This case suggests that as a mechanism of governance, or a productive technology, the HMIS program organizes and makes available for intervention a homeless population that exceeds the individual, but in which the individual is caught up. The privacy concerns raised by critics are not irrelevant, and they have played a role in shaping the universal data elements. But ultimately, the protections provided do not attend to a form of politics operating on a register other than the individual subject with a claim to privacy. Confronting the politics of homeless population surveillance requires concerns beyond individual rights.

To understand the implications of HUD's HMIS program requires thinking about the technical form of the systems. To do so, the next section situates HMIS in a history of number-gathering technologies and practices and explores what it means to think of the universal data element as a live and active connector between databases and networks.

\section{The Database and the Network}

While certainly many other technologies are at play in contemporary social services directed at homelessness, the information management demands that characterize contemporary governance depend upon the organizational capacities of the database and the circulating capacities of the network. Exploring the interaction of these two technological forms helps elucidate the significance of HUD's HMIS program. Again, as HUD has emphasized, its HMIS program does not create a national database of homeless people. Nor does it control the structure of those databases or all the kinds of data sharing that take place. Rather, it facilitates the creation of agency databases and the sharing of data between agencies. Despite "giving up" much control to municipalities and agencies, HUD nonetheless is able to achieve the formidable task of centralized data collection in a 
post-welfare state context. Its HMIS program outsources the labor of producing raw data to the various agencies it funds while, by legislating the form and content of that data, allowing for the execution of a centralized state function-the monitoring of a population at a national level-within the void of a central welfare state. From one angle, HUD's program seems to almost produce a national population in the absence of a nation.

We should pause to consider what is meant by "database." Most generally, the database is a system for the classification, organization and retrieval of heterogeneous materials or elements (Manovich, 2001). In this sense, it is not the first such system; various other methods have obviously existed for quite some time (Bowker and Star, 1999). Eugene Thacker notes dictionaries, encyclopedias, anatomical atlases, maps, and statistical charts as the forebears of the contemporary computer database (Thacker, 2006:105-106). Given the social service context in which we are considering the use of databases, another obvious historical precedent is the filing cabinet stuffed with manila folders, containing everything from case notes, to identity documents, photographs, medical records, and copies of bureaucratic forms related to health benefits, income support, and legal proceedings. All of these systems employ a specific method of organization that "depends on the technical specificity of the system; whereas the dictionary and encyclopedia utilize an alphabetic system, the anatomical atlas utilizes a diagrammatic logic, and statistics utilizes a series of tables, charts and quantitative graphical elements" (ibid.).

These systems generally are understood as a means to organize various elements into some sets of relationships, particularly with an eye towards ease of retrieval. However, thinking in terms of logics of organization reminds us that each system offers constraints on not just the organizational mode, or sets of possible relationships, but also on the form of the compositional elements themselves. In a dictionary, while an illustration of an "apple" can supplement a textual description of the word "apple," it cannot stand on its own. The illustration's lack of an alphabetical attribute denies its entry into this system, except as back-up for the word-element "apple" (which possesses an appropriate attribute, beginning with the letter "A"). A statistical chart that displays how many respondents to a survey are White, Black, Asian or Hispanic cannot include the narrative descriptions of family lineage, ethnicity, and immigration that individuals commonly use to describe their "race." Such narratives must be operationalized, or assigned attributes that make them organize-able within this system, otherwise they cannot materialize in the chart. Finally, while the copy of a summons for creating a public nuisance can easily find its way into a social worker's manila folder, warmly tucked between case notes and a birth certificate, a plate of food refused in protest of the police at mealtime cannot. This is to say, the refused meal cannot enter into the official record, as the structure of organization (a folder within a system of folders) cannot accommodate such an element. So while the computer database is not the first system of classification, organization and retrieval, it is, as others have pointed out, uniquely capable of integrating any number of other systems into itself: the database can organize and present elements as alphabetical lists, as statistical graphs, as a series of illustrations. This occurs through a process of digitization, or the translation of the raw material into binary form.

The kinds of data that HUD generates through its HMIS program are not unique to this historical moment, but belong to a long history of number-gathering and its evolution into 
the modern statistics of which HUD's HMIS program is a part (Scott, 1999; Cohen, 1999; Porter, 1995; Hacking, 1990). While gathering numbers predates the rise of the modern state form, statistics (the etymology refers to "state data") marks the shift from a gathering of numbers for numbers sake (as in pre-modern Parishes in Sweden, as Ian Hacking notes) to the production of numbers put to work, and as such, is central to the rise of the nation state. This work might solidify as natalist policies, electoral districts, unemployment forecasts or insurance rates, to name a few governmental uses of statistics (Porter,1995). At first glance, HUD's HMIS program could seem to be simply an extension and perhaps intensification of the "avalanche of numbers" (Hacking, 1982) that has washed across the United States since the imperialist invasion of the Americas. But some important shifts are provoked by the use of the database. If governmental numbergathering has always been activated, here we have a kind of data-which is to say, digital data - that realizes that action component in its very form. A database is not simply a collection of numbers, but it is numbers constructed in relation to action-the algorithmic functions of the computer, or, more specifically, the database management system (DBMS). This is then to recognize that while other systems of organizing heterogeneous materials (dictionaries, atlases, filing cabinets) may have struggled with how to allow efficiently for the retrieval or reorganization of those materials, the computer database organizes matter in the form of matter-set-to-retrieval. In fact, the computer database departs from older systems in that referring to a process of retrieval is something of a misnomer. Within a database system, the process of retrieval is very often actually a process of generation. The DBMS allows for the production of data, not simply its organization and retrieval. While a dictionary can only contain those elements entered into it (even, say, an online dictionary that can be endlessly revised), a database makes more than what was already there, the raw data input to begin with. In computer science terms, this is to say that what might be "processed data" at the end of one algorithm is available to become "raw data" for another set of computations, or retrievals. Data entered into a database should not be thought of as fixed or stable. Data are mobile points of connection that facilitate the dynamic algorithmic functions of a computer: organizing data into new combinations, new relationships and, most importantly, new calculations to make new data itself. The configurability of HMIS applications is geared toward this data generation process. The universal data element becomes a raw material available for use by HUD, state and city governments, Continuums of Care, and service agencies.

But the magic of the universal data element is that its productivity is not confined to its database of origin. The universal data element can travel, between platforms and across locales, while retaining its generative capacities. This is to say that HUD's data standards assure that any universal data element can be put to work in any new HMIS database context, as it will be commensurate with the native elements. Thus data standards can be thought of as embedding networking capacities within universal data elements. In other words, elements produced in different locales, through different databasing technologies, can be connected together, and set to new relationships.

Thus universal data elements can circulate through any number of networks. These networks may be the literal distributed networks of internet-based HMIS applications used by an agency, or a closed network drawn from this distributed network, between agencies constituting a Continuum of Care. They may also be "social networks" of service providers and governmental officials. And they may need to travel through such 
old-fashioned networks as the U.S. Postal Service, as agencies mail compliance reports to HUD. I take seriously Thacker's warning against the metaphorization of "network," such that anything today can be taken as a network and the actual material structure of networks is vaporized into a vague sense of "interconnectedness" (Thacker, 2004:xii-xiv). In fact, the case of the HMIS program involves a great variety of networks, some of them the variously structured technical networks of computer science, and some of them social networks involving organizations and individuals. So we could say that an HMIS is involved in networks of networks, and that HUD's program instantiates a socio-technical network (constituted by funding streams and the mandates that follow from them) under which all the networks at least partially lay. I do not wish to point to "the" network that constitutes the governance of homelessness, but rather point to the capacity of the universal data element to move between any number of DBMSs and any number of networks. This is what I meant earlier when I said the universal data element acts as a connector between the database and the network-it allows these two technical systems to co-function, and for each to benefit from the capacities of the other: the database classifies, organizes and generates data that is given up to the network; the network carries that data beyond the confines of the DBMS.

HUD's HMIS program is an exemplary case of neoliberal or post-welfare state governance in how it permits the state both to disperse and contract. This networked database system allows for any number of nodes, or agencies, to operate independently within a structure that is decentralized but nonetheless subject to parameters or controls. This is much more powerful than a centralized state, or a centralized database. The network is mutable and can survive even when nodes in the system fail; for example, one local jurisdiction's bad data would not stop the flow of data to HUD overall. Furthermore, the labor of generating data does not need to be centrally organized or coordinated by HUD. The use of universal data elements produces commensurate materials that can both operate at local levels (within the agency and its target constituencies, as well as in local Continuum of Care jurisdictions) and at national levels. At the local level, the data can be attached to a unique client, and can therefore serve an agency's work of case-by-case monitoring. At the national level, the data sheds its unique identifiers and becomes part of an aggregate, thus satisfying claims for privacy and drawing together to form a national count, which is to say, the universal data elements organized by each local database can be used to generate other data that can be processed again into the shape of a national count that Congress demands. This aggregation of data can be thought of as something like the collectivization of labor that produces more than the sum of each laborer. And I will argue that "the population" is the surplus of the collectivization of the universal data elements.

If the above describes the technical capacities of an HMIS, what might be some implications of using an HMIS for homeless social service provision? In the next section, I will explore both enthusiastic endorsements and cautionary warnings regarding the electronic turn in social work. While offering an overview of responses to HMIS and related technologies, this will help situate HMIS in a history of technology in social work. It should also expose some of the questions that neither technophilic nor technophobic positions can pose. 


\section{The Electronic Tum, For and Against}

The Senate report cited at this article's open expresses dismay at what was a fifteen-year lapse in federal oversight of homelessness initiatives, a lapse which would seem symptomatic of social service provision after the welfare state. With services provided by such great numbers and kinds of agencies, the federal government's capacity to monitor and assess these programs has faced obvious challenges. From the technical perspective of meeting its federal mandate, the dilemma facing HUD can be posed as: How can data generated at dispersed contact points not under direct federal control be centrally organized?

HUD argues that the use of an HMIS benefits not just the federal government, but also agencies providing services. In language familiar within a general turn towards quantitative models in health and social services (Clark et al, 2003; Strauss and McAlister, 2000), HUD argues that an HMIS allows a variety of tasks to be completed more efficiently and accurately, "bringing the power of computer technology to the dayto-day operations of individual homeless assistance providers" (Office of the Federal Register, 2004:45897). According to HUD and other advocates of the electronic turn, the first set of tasks an HMIS improves upon are related to service provision, and involve the use of standardized intake and assessment procedures. This is meant to replace the largely "anecdotal" methods of earlier eras of case management. Rather than each caseworker developing an individualized, subjective assessment, one method of accounting for something like "disability status" is developed. Thus caseworkers utilize commensurate measures that allow for the translation of one worker's notes to any other worker and eliminate the labor of producing those measures case-by-case. Additionally, HUD understands these measures as making for better collaborative work across agencies, which it describes as "[k]nitting together housing and service providers within a local community into more coordinated and effective delivery systems . ..” (ibid.). This can take place because unique identifiers such as name and social security number can be shared between agencies, just not with HUD. Thus an HMIS allows for the tracking of clients utilizing multiple agencies within a Continuum of Care in a local community. HUD offers the example of an agency being able to determine if a client requesting service has already received that same service from another agency. While this clearly serves to police the poor from "double-dipping," HUD sells it as a way for agencies to better allocate resources where they are needed and useful.

In addition to benefiting service provision, the use of HMIS is understood as creating more accurate "pictures" of homelessness, which both serve agencies and allow HUD to produce an unduplicated count of the national homeless population. Thus HMIS produces a more detailed and accurate picture of homelessness at both local and national levels:

HMIS can help local communities understand how many people are homeless in shelters and on the street; how many people are chronically or episodically homeless; the characteristics and service needs of those served; and which programs are most effective at reducing and ending homelessness. 
HMIS can help HUD and Congress understand: how many people are homeless in the United States; who is homeless; where people receive shelter and services and where did they live before they became homeless; the patterns of homeless residential program use; and the nation's capacity for housing homeless people (U.S. Department of Housing and Urban Development, 2006b).

A presentation prepared by Abt Associates for HUD's use in "recruiting” agencies to HMIS explains that "HMIS is a tool, not the goal." This phrase is meant to show that HUD is not attempting to change what service providers do, only help them along (with funding attached to the requirement the agency use an HMIS-a coercive, paternalistic help that seems to have survived the death of its father, the welfare state). Comparing service provision and counting methods with and without the use of an HMIS, the presentation demonstrates how HMIS not only accomplishes what traditional methods cannot, but also better realizes the accomplishments of older methods. According to this presentation, traditional service provider reports are not able to produce unduplicated counts (as the same client may be counted as a unique individual at two or more different agencies) and not able to assess patterns of "entering and exiting homelessness" (patterns here understood to be statistical portraits). On the other hand, not only can HMIS accomplish these two goals, it can also capture "in-depth information on clients and needs," the one accomplishment accorded traditional, narrative-based service provider accounts. HMIS is able to do this through user configurability, such that areas of need or service specific to an agency can be tracked with HMIS, in addition to the data required for producing the compliance reports containing an agency's summary of universal data elements. From this perspective, HMIS offers the best of both worlds: it captures the universal (universal data elements) and the particular (the specific data interests of agency and locale). Thus, HMIS allows for accurate counts of homeless populations; adds a longitudinal dimension of patterns over time; and (contrary to critics, whose arguments are explored below) preserves the richness of caseworkers' understandings of clients and their needs though an amassing of quantitative detail.

Critics of HMIS, and social work's electronic turn in general, take another view of what databasing social services can and cannot accomplish. While HUD's discussion plays out familiar technophilic themes-HMIS makes things easier and faster, saving money and time-critics suggest that HMIS is not simply an enhancing "tool," but rather a forceful intruder that endangers the values and practices of social work, recapitulating technophobic themes that warn against handing control over to "the machine." To appreciate these concerns, we should note how social work understands that an intersubjective relationship between client and caseworker forms the foundation from which social service provision can most effectively operate. This intersubjective relationship came to social work via psychodynamic psychiatry and its asylum-based instantiations, from which the case management model of service provision drew inspiration. Psychiatry offered the conception of the client as a "case" with a unique history and background that could be best accessed and understood through an intersubjective exchange between caseworker and client (Webb, 2006:111-117). Thus social work emerged as a hermeneutic art dependent upon a set of tools-face-to-face conversation, detailed biographical notes, and archives of the seemingly endless 
documentary detritus of modern bureaucratized existence-that meant to form a full picture and clear understanding of how the case came to be in its current state of need. The relationship between worker and client offered the means to assessing and assisting the case. ${ }^{7}$

By the late 1960s or early 70s, this relationship became insecure, as psychiatric-based theories and practices came under fire (Webb, 2006:121-125). The inability of psychodynamic practices to produce cures in patients, especially as evidenced by the growing and burdensome indigent populations of federally-run mental health asylums, offered an opportunity for the rise of neuroscientific models that located mental illness not in the history of the case, but in the misdirected activity of the brain (Shorter, 1997; Dowbiggin, 1997). The erosion of psychodynamic authority helped introduce to social work the pressures concomitant with the rise of evidence-based medicine, in particular the requirement to produce the "hard science" of numbers about diagnosis and treatment.

Thus, the liabilities that HUD understands the use of HMIS as correcting - the ad-hoc, interpretative, anecdotal practices of the well-meaning but un-scientific social workercritics of the electronic turn understand to be precisely the unique resource that social workers offer, a resource threatened by the use of machines. Bob Sapey, for example, draws from studies of the relationship between decision-making and communication media to argue that the complexity of the social worker's milieu demands the "richness" of interpersonal conversation:

... [M]essages which are sensitive or complex and which require a greater degree of explanation to be understood, will require a medium that will permit more direct interaction between the sender and receiver. The richest medium is generally face-to-face talking, in which non-verbal communication can be incorporated and there is opportunity for response and exploration of the issues (Sapey, 1997:810).

Thus the depth offered by psychodynamic casework contrasts with the superficiality or surfaciality of electronic data. As the interpersonal relationship gives way to an impersonal information exchange, the electronic turn instates an evaporation of the multidimensional knowledge structures of social work. Technologies such as HMIS are understood to intensify, if not enact, a rationalization and instrumentalization of knowledge.

Identities are constructed according to the fields that constitute the database, so that in striving for clear and objective representations and decision making, the subjectivity and social context of the client can be deconstructed into a variety of lists and factors associated with, in particular, "need" and "risk." Categorical thinking, based on the binary either/or logic, dominates, which puts individuals into categories and, in the process, obscures any ambiguities. Rather than be concerned with presenting a picture of the subject, as previously, social work

\footnotetext{
${ }^{7}$ Critiques of medical models in homeless service provision are explored by Snow et. al., 1994 and LyonCallo, 2004. Some of the political uses of medical models, particularly legal disability status, are suggested by Blasi et. al., 2007.
} 
increasingly acts to take subjects apart and then reassembles them according to the requirements of the database. Practitioners are required to produce dispersed and fragmented identities made up of a series of characteristics and pieces of information which are easy to input/output and compare. In the process, the embodied subject is in danger of disappearing and we are left with a variety of surface information which provides little basis for in-depth explanation or understanding (Parton, 2006:11).

Critics implicitly reject HUD's insistence that databasing social work can preserve the richness of the social worker's understanding of clients. In the quotation above, not just the caseworker/client relationship, but the client him or herself, is eroded by the use of computer technologies. The amassing of data that HUD argues adds up to a complete picture, from this view is simply a quantitative increase in superficial content (Aas, 2004).

While these debates about the electronic turn play out familiar tropes in technophilic/ technophobic discourse, as well as conversations in the social sciences about the advantages of either quantitative or qualitative methods, both positions ultimately fail to recognize the place of the technological in the social generally, and social work specifically. Keeping in mind the argument that HUD's program depends upon the generative capacities of databases and the circulating capacities of networks, the next section considers the productive capacities of technologies in social service provision.

\section{The Productive Capacities of Technology}

From a technical perspective, it is fairly easy to point out the fallacies embedded in HUD's promotional materials for HMIS. In pushing the advantages of HMIS over older forms of case management, HUD overstates the fail-safe aspects of this technological form. While HUD contrasts the clean data produced by an HMIS with the subjective and incomplete accounts of case managers and "one-night counts," database programs of course also fall short or fail-systems crash, networks go down, files get mysteriously deleted. HUD also promotes HMIS as if the information it generates is transparent, or will simply "analyze itself," rearticulating a mythology of digital information as instantaneous and self-contained. But in fact the aggregation of data requires quite extensive and complicated record keeping and reporting to generate the numbers and make them work. It also requires plenty of extra-database, human-based computations and analysis, including seemingly endless forms upon forms that agency staff must manually complete.

But more interesting perhaps is to note the blind spots in arguments against the electronic turn in social work. By basing critiques on the intrusion of the computer itself, critics fail to consider the long history of the technological configuration of social work practices. When past technologies are accounted for, their role is understood in very much the sense that HUD understands HMIS - as a tool taken up by the social worker in support of the central, intersubjective tasks. For example, Nigel Parton points to a set of technologies central to social work prior to the electronic turn, from the 1950s to the 1970s: 
While "the relationship" was the primary tool or "technology" of the work, there were also a variety of other technical devices that were drawn upon so that individuals would be rendered as knowable, calculable and administrative "subjects." These devices were in the form of a variety of written reports but also included other devices, such as the car and the telephone, which made it increasingly possible to negotiate time and place more efficiently and quickly (Parton, 2006:6).

Here Parton offers an important analysis of the technical apparatuses that made earlier models of case management possible, and recognizes the interpersonal relationship itself as a technological form (scare quotes notwithstanding). However, this analysis, unfolding within a technophobic reaction to computer-based technologies, renders the written report, the telephone, the car, and the relationship less technological and therefore less dangerous. Thus Parton argues:

What becomes evident is that, increasingly, such [technological] developments not only acted to support and refine the work, but become a major influence in reconfiguring the form of knowledge itself. Such developments become increasingly evident with the introduction of new systems of information technology (IT) and, more recently, ICTs [information and communication technologies] (ibid.).

The ways in which computer technologies make the technological configuring of social work "increasingly evident" is mistaken for evidence of social work being increasingly technological. A guilt by association is projected onto practices that rely on computer information technologies, producing in turn a foil of innocence for those practices that relied on "tools" thought to be less-technological and more genuinely human/humane.

But, after Michel Foucault, we know that there is no form of knowledge absent a "technical substrate" (Clough, 2000): knowledge is always bound to the technologies that allow for its registration and distribution. The written report, the car and the telephone did not simply aid the intersubjective relationship between caseworker and client, they made it possible, and did so through the production of the client as a case: an individual subject technically open to intervention, which is to say, available to be studied, known, and treated. Foucault's work suggests that the development of social work practices and the subject positions embedded within them-the caseworker and the client-might best be understood within a broader development of the sciences of man, such as psychology and its related fields, especially sociology and criminology (Foucault, 1994). In his study of one technology central to the emergence of the sciences of man and its accompanying disciplinary management techniques — the photograph-John Tagg writes:

Whatever the claims of the traditional evaluations of such photographic "records," whatever the pretensions of the "humane" and documentary tradition, we must see them now in relation to the "small" historical problems with which Foucault concerns himself: problems of the entry of the individual into the field of knowledge, of the entry of the individual description, of the cross-examination and the file. It is in what he calls these "ignoble" archives that Foucault sees the emergence of that modern 
play over bodies, gestures and behaviors which is the emergence of the socalled "science of man" and the constitution of the modern state (Tagg, 1993:92).

Tagg reminds us that a photograph is never only a record, but is always productive, and not simply of knowledge, but of the object to be known and the relations of power that criss-cross through that object. Thus, the knowledge of the caseworker, the loss of which cautionary tales currently bemoan, is not simply an heuristic position that offers depth against the gleaming surface of digital transfers, but is in fact a technology that binds together the individual subject - the case-and draws that subject into relations of what we must think of as disciplinary management. From this perspective, we can understand case management as a technical apparatus of disciplinary power, and the humanism that critics of HMIS worry is being undermined by the electronic turn can be understood as, rather than a palliative program of assistance, a constitutive political project. ${ }^{8}$

Social work is always already technologized. The use of technology in social work neither enhances nor degrades a core or true practice of the service provider, but rather, the act of service provision is a set of technical operations for the disciplining of the subject. $^{9} \quad$ Recognizing this moves us toward an understanding of technology as productive, or constitutive. Here I do not mean to fall into technological reductionism or social constructivism, but to point to the indistinguishability of the technological and the social when we think both in terms of the organization of matter toward its openness to intervention. As I will explore in the next section, the interdisciplinary field of surveillance studies usefully takes up the productive capacities of the social and technological. While this literature often picks up on anticapitalist technophobic themes, it also draws attention to what information technologies of surveillance create and make possible, not simply what they corrupt (Monahan, 2006; Haggerty and Ericson, 2006). This is to think of how surveillance technologies organize possibilities and kinds of living, rather than simply record life as it is. Most specifically, surveillance studies has looked to the productive capacities of information technologies, and notes how technologies of surveillance are not simply tracking individuals, but actively generating its object of intervention-data. In this sense, surveillance technologies are not seen just as repressive (controlling people and their movement), but productive of something new.

\section{From Disc iplinary to Biopolitical Surveillance}

In an influential article, Kevin D. Haggerty and Richard V. Ericson designate an emerging "surveillant assemblage," or the coming-together of once independent systems of surveillance. One result of this new assemblage is the production of what they and others call the "data double":

Today ... . we are witnessing the formation and coalescence of a new type of body, a form of becoming which transcends human corporeality and

\footnotetext{
8 For an excellent account of disciplining mechanisms and subject-production in a homeless shelter, see Lyon-Callo, 2004.

9 The classic study of social welfare programs as systems of social control is Piven and Cloward, 1993.
} 
reduces flesh to pure information. Culled from the tentacles of the surveillant assemblage, this new body is our "data double," a double which involves "the multiplication of the individual, the constitution of an additional self” (Poster 1990:97). Data doubles circulate in a host of different centres of calculation and serve as markers for access to resources, services and power in ways which are often unknown to its referent (Haggerty and Ericson, 2000:613).

Here, Haggerty and Ericson offer an alternative understanding of information technologies such as HMIS. Pointing to not only the diffusion, but also the linking up, of surveillance technologies across the social, they argue that the surveillant assemblage does more than "spy upon" past actions, as is often the expressed fear of surveillance technologies. Rather, these technologies orient toward the future, determining the distribution of access to resources and life chances in arenas of health, education, employment, consumption and civic life.

I want to push this analysis a bit further. To argue that the data double "serves as a marker" for access does not say strongly enough how information technologies open and close channels through which possibilities of life itself circulate, as the case of HUD's HMIS program will make clear. The marker is an active agent, drawing capital and bodies towards it. And I would say that not only does this happen in ways unknown to the data's referent, but in fact, in its circulation and especially in its form as statistical probabilities, data becomes divorced from its referent and takes on a life of its own. Statistical data that composes the consumer and risk profiles Haggerty and Ericson and other scholars of surveillance discuss derives from the calculation of aggregates that exceed an individual referent, such as HUD's population counts. This is to point to the existence of a field of data of which any one individual is only an incomplete compositional piece, but in which all individuals are caught up. This is also to challenge the assertion that surveillance data form "an additional self" or a "new type of individual" (Haggerty and Ericson, 2000:614). This concept of "data double" hovers too closely to the individual subject, reducing data to a shadow, or a reflection, of a specific person, and therefore cannot account for what is produced by the technology above and beyond the scale of an individual body/subject.

In other words, the data double has been chained to the object of disciplinary management - the subject's body and its movement in space. To capture the sense of a field of data as I describe requires looking to what Foucault called the other pole of biopower, biopolitics. Disciplinary management, including the psychodynamic model of case management in social work, describes those techniques of power organized through disciplinary enclosures and directed at the human subject, the body and soul of that subject, and its relationship to other subjects in space. Biopolitics, on the other hand, seeks to regulate the social or collective processes of life, death and productivity across a population (Foucault, 1978:139; See also Foucault, 2003:239-264). Together, discipline and biopolitics function to bring biological objects and processes into political and economic calculation; discipline does so by addressing the animal body of individualized man whereas biopolitics does so by addressing the species body of the total population.

HUD's effort to produce a national homeless population, then, must be considered in 
terms of a biopolitical project that disciplinary models of surveillance studies cannot fully describe. In fact, the historical situation described earlier as neoliberalism or the withering of civil society can be thought of as circumstances in which this second trajectory, biopolitics, becomes predominant. In this post-disciplinary context, discipline does not fall away. Though welfare state institutions begin to lose distinct form under internal and external pressures, this does not mark the disappearance of techniques of social control associated with schools, hospitals, prisons and the lot. Rather, accounts suggest that these techniques have become unmoored from their institutional arrangements, allowing for a more general distribution of abstract disciplinary practices across what had been known as the social. Further, in the context of the coming-todominance of biopolitics, the remains of disciplinary enclosures may begin to serve new functions. The opening up of the enclosures, their multiplication and diffusion, suggests that the organization of the individual and its confinement in space are no longer the primary goals of disciplinary mechanisms. Rather, disciplinary mechanisms in this context may serve as flashpoints between an individual and biopolitics; as HUD's emphasis on data collection and data quality suggests, it is the individual set in relation to data that matters most. The technologies of disciplinary psychodynamic case management-including the intersubjective relationship of caseworker and client as well as institutional arrangements of non-governmental agencies, service plans and federal mandates-provide a track across which biopolitical techniques of information management can roll out. Tossed into an empty field with nothing to feast on, HUD's networked system would curl upon itself and die from autophagy. But inserted into existing technological configurations of homeless management, HUD's information system feeds on the techniques of discipline perfected in social service provision and nourishes them in return, keeping case management dependent but alive through symbiotic arrangements of mutual parasitism. In its withering, civil society does not disappear... it is absorbed in a weakened state into a biopoliticized milieu in which its practices continue to reproduce, though in mutated form. Inside this new milieu, the management techniques of civil society become viral bodies, distinct yet inseparable from the larger milieu-body, forceful yet dependent upon their contextual embeddeness for continued existence.

So it is for social work after the electronic turn. HMIS draws disciplinary homeless social services into an information management milieu that the database, the network and social work together engineer. And so social work is neither simply enhanced nor eroded by a technology it deploys, but rather is itself deployed inside this technically-configured context. In order to survive, social work must transform itself to move within the contours of this new milieu, and that survival may be in a form difficult to recognize. Though these disciplining techniques may continue to accomplish some of their older goals, they may also survive insofar as they hold the subject available to biopolitical intervention that will in turn leave the subject behind.

The consequence, then, of surveillance studies remaining at a disciplinary register is not that its analysis is wrong, but that it can't fully account for the productive operations of power at play when it assumes an individual subject as the object of intervention. The universal data elements function at the level of a population organized by a sociotechnical apparatus of federal agencies, social policies, service providers, databases and networks. The surplus created by HMIS is a homeless population in excess of an 
individual subject. This population of universal data elements is then set loose on those individuals who have provided its raw material. This is to emphasize not only that we think in terms of populations rather than individuals, but to recognize the populational as a register that must be constantly produced as a site of intervention. In other words, just as discipline must engage in perpetual processes of interpellation (the "self" is not made once, then plunked down into a body), the technologies of biopolitics must constantly calculate and recalculate what counts as a population and how it is counted in order to remain vital means of biopolitical management. The population must bend to the contours of the mutability and unpredictability of the life itself of the human species. The population does not precede the technologies that count it: biopolitics manages the population by organizing the biological species as a population plane of intervention. Whereas HUD would imagine the "unduplicated population counts" that its HMIS program makes possible as simply a statistical reflection of what actually exists, we can rather understand HUD's program as arranging homelessness as a population, and therefore as vulnerable to the biopolitical calculations of its HMIS program. This is not a population as a representation of "the people," but rather a plane of intervention formed from the raw material of homeless clients that draws bodies back into itself. The answer to critiques that HMIS signals an invasion of privacy has been the creation of "masked data" that cannot be traced back to a specific client. But whether or not a specific individual can be related back to data generated out of that individual, the life of that data will absorb and transform the life of that individual. The population is a living entity injected with biopolitical force that acts back upon that which made it.

\section{The Biopolitic ization of Homelessness}

Thus HUD produces a homeless population that is a population of universal data elements cut off from the subjects from which they were mined. The population does not reflect the individuals that made it up, but the population works toward "making up people," to play with Hacking's formulation (Hacking, 2007). If Hacking means to point to how the categories of statistics determined what kinds of people could be conceptualized (what kinds of laborers, for example, or that people would be categorized in terms of the labor they performed at all), I want to point to how the production of populations makes some kinds of living possible, while foreclosing others. To conceive the power of the population as a register for intervention in biopolitics, we must remember to think of the population, like the data that generates it, as an active element, and not as simply a representation. The population changes, and provokes change. As argued above, in its forceful mutations, it is oriented not to the past (the time-lag between the reality the data is meant to reflect, and the solidification of the count) but rather to the future. The data that is generated by HUD also travels along network channels, and in this return, distributes life chances and health opportunities by organizing relationships between governance, services, resources, and bodies folded into a target population. The homeless are made by this population data, which is to say, the data determines what kinds of life are made available by programs targeting the homeless.

To think the population slides us right into to thinking governance, and they might be thought of as two different angles of the same conglomerations. To evoke the population 
is to already say you are dealing with biopolitics (as to evoke the individual is to say you are dealing with discipline). But there is a unique form of governance emerging here, specific to a history of post-welfare homeless management in the United States (though certainly similar turns are taking place elsewhere ${ }^{10}$ ). The State of Arizona provides a useful example of how data generated through HMIS is put to use toward the production of not just a population, but governance itself. For agencies receiving its McKinneyVento Act funds, Arizona has developed what it calls a "self-sufficiency improvement score” (U.S. Department of Housing and Urban Development, 2006a:22). In terms of the clients of service agencies, this score accomplishes two things. First, it creates a standard measure of "self-sufficiency," thereby operationalizing what perhaps in other eras have been vague and subjective outcomes; now a number can be attached to a client's ability to care for themselves. Secondly, it allows their progress over time with an agency to be measured, giving a longitudinal dimension to this data. The self-sufficiency score serves as a good example of exactly the concerns raised by critics of the electronic turn, and it will also show concerns that go beyond theirs. The self-sufficiency improvement score represents an amalgamation of various areas of need and service but also their quantification. The subjective sense of being able to care for oneself, as well as the caseworker's sense of this, here is eclipsed by a numerical measure. This is the client decomposed and recomposed as data points, as described by critics earlier.

However, HUD's commentary on this program makes clear that how this data will be used to serve clients is of almost incidental concern. The State of Arizona plans to use this data to create aggregate rates of self-sufficiency improvement for agencies. Thus, Arizona can compare and rank agencies, and funding can be directed at them based on their successful generation of data that improves. HUD describes this as "performancebased contracting" (Ibid). For agencies, making their numbers "get better"-not necessarily their clients-will draw in more money. Improving rates of self-sufficiency may not be the same thing as improving the lives of homeless people in Arizona. Here we can see the quick movement from an individual who might look something like a case, to their reconfiguration as a data profile of numerical scores, to their absorption as part of a larger data body (clients of an agency) out of which is generated numbers to draw in funds. If anything, HUD here is successfully regulating agencies, not clients, and it is doing so through "flexible regulation." Arizona operates within a given set of parameters (the gathering of universal data elements by each agency supported with HUD funding) that can be actualized in any way the State of Arizona deems appropriate. It is "free" to make other uses of HMIS, and it has done just that. By providing flexible limitations, HUD allows states, cities and Continuums of Care to innovate. They thereby function as test laboratories, producing new uses of HMIS that HUD can circulate as models, to be repeated and further elaborated upon by other locales.

The clients become a way of determining where money, resources, and technical information will flow, and what the life of an agency might be. This could be thought of as a governance of governance that operates through disciplined bodies mined for population data. And the population generated (a population of agencies, overlaying a population of Arizona homeless) feeds back on those disciplined bodies, the raw material

\footnotetext{
10 Mass incarceration, whose populations interact and overlap with homeless populations, might be the other exemplary case of US neoliberal biopolitics. See: Gilmore, 2007.
} 
out of which this data generation takes place. So, for example, an individual in Arizona will find the service options that become available to him or her will be dependent on (1) how that individual is sorted within the general population (as someone with chemical dependence or psychiatric disabilities for example) and (2) how population data for that track has determined what agencies exist for that track and what services they offer.

The use of HMIS data by the Washington D.C. Continuum of Care offers a further example of the valuable surplus generated by universal data elements, as well as practices of "governance of governance," or the use of a homeless population to regulate service agencies. Working with the Department of Health and Human Services, the D.C. Continuum of Care has developed an "Annual Performance Plan" that all D.C. agencies receiving McKinney-Vento funds are required to submit. These plans are used to evaluate agency performance, and to help determine how D.C. will allocate its McKinney-Vento funds. As a report on this initiative notes:

The ability to use HMIS data for more than required reporting is a tremendous asset to the CoC [Continuum of Care]. The D.C. Performance Measurement System has enabled CoC staff to collect data on each program's performance and monitor progress toward individual program and broader community goals. As shown ... . the Annual Performance Plan is a well-defined, structured way in which to hold providers accountable and ensure everyone clearly understands what is expected (U.S. Department of Housing and Urban Development 2007b:28).

Three accountability factors are tracked by the Annual Performance Plan using HMIS generated data: the occupancy rate of the program; the income clients obtained while in the program (including public benefits); and either the successful transition to or maintenance of permanent housing. These three factors are used to create comparative quantitative measures of agency performance, which are in turn used (though not solely) to determine D.C.'s funding priorities. While we do not want to misread this as some sort of automated technocracy that matches dollars to scores, we should note what a far cry this use of HMIS is from HUD's promotion of it as a way to do what agencies already did, only better and faster. These three measures, which translate client experiences into agency scores, operationalize better and faster as injunctive commands that agencies must respond to in order to remain competitive in a context of scarce and restricted funding. ${ }^{11}$ Individual experiences of finding and maintaining appropriate shelter, or accessing income necessary for survival, are absorbed into a governance mechanism in which the "regulation of the poor," to borrow Piven and Cloward's phrase, functions not only as social control, but also as a mechanism for the regulation of social services. Being a target of homeless social services here becomes a kind of labor used to produce a population for governance and as governance.

\footnotetext{
11 In pointing to the regulation of homeless service agencies, I do not mean to endorse their activities, or suggest they should not be regulated. I mean only to point to how the productive capacities of universal data elements are used for many purposes well beyond the counting or even tracking of homeless individuals. The professionalization of social services, the use of social services to extend state power, and the role of social services in maintaining inequality all remain to be explored in this context. Such issues are taken on in relation to homelessness by Lyon-Callo (2004) and Funiciello (1994), and more broadly by Piven and Cloward (1993) and INCITE! Women of Color Against Violence (2007).
} 


\section{Conclusion}

The cases of Arizona and D.C. indicate what it means to say that targets of homeless governance are caught in a field of data not tied directly back to themselves, but to which they are very much bound. HUD's HMIS program produces a population of universal data elements that circulates powerfully in the biopolitical, post-disciplinary context of contemporary governance. Contrary to HUD's claims, this population does not merely present an accurate picture of homelessness in the U.S., but it rather re-makes homelessness by reconfiguring what needs are allowed to register, and what services can address those needs. In fact, the population precedes the lives that made it, as the individual becomes drawn into the possibilities of life determined by biopolitical technologies. The individual must come to reflect the population, not the other way around. Providing privacy protections for individual clients - as critics demanded and as HUD has done-does not shield that individual from such feedback effects of this population data. Surveillance technologies in this context do not "clamp down," but rather let loose. They let loose a biopolitical register toward the proliferation of governance and its populations, which is to say, they free new objects of intervention that may not look like any one individual, but of which the individual client, in order to survive, must become a part. The client gives life to the population, and receives life in return: the bare minimum of food, shelter and healthcare exchanged for offering oneself up as raw material for the productions of HMIS. Within biopolitical homeless management, for individuals in desperate need of resources controlled by social service agencies, there may be no life outside the life given by the population.

\section{References}

Aas, K.F. (2006) From narrative to database: Technological change and penal culture. Punishment \& Society 6: 379-393.

Blasi, G. and The UCLA School of Law Fact Investigation Clinic. (2007) Policing Our Way Out of Homelessness? The First Year of the Safer Cities Initiative on Skid Row. Available at http://www.law.ucla.edu/docs/policingourwayoutofhomelessness.pdf

Bowker, G.C. and S.L. Star. (1999) Sorting Things Out: Classification and Its Consequences. Cambridge, MA: The MIT Press.

Burchell, G., C. Gordon, and P. Miller (eds.). (1991) The Foucault Effect: Studies in Governmentality. Chicago: University of Chicago Press.

Clark, A.E., J.K. Shim, L. Mamo, J.R. Fosket, and J.R. Fishman. (2003) Biomedicalization: Technoscientific Transformations of Health, Illness, and U.S. Biomedicine. American Sociological Review 68(2): 161-194.

Clough, P.T. (2007) Introduction. In P.T. Clough (ed.) The Affective Turn: Theorizing the Social. Durham: Duke University Press: 1-33.

Clough, P.T. (2004) Future Matters: Technoscience, Global Politics, and Cultural Criticism. Social Text 80 (22:4): 1-22.

Clough, P. T. (2000) Autoaffection: Unconscious Thought in the Age of Teletechnology. Minneapolis: 
University of Minnesota Press.

Cohen, P. (1999) A Calculating People: The Spread of Numeracy in Early America. New York: Routledge.

Deleuze, G. (1992) Postscript on the Societies of Control. October 59 (Winter): 3-7.

Dowbiggin, I. (1997) Keeping America Sane: Psychiatry and Eugenics in the United States and Canada, 1880-1940. Ithaca: Cornell University Press.

Foucault, M. (2003) Society Must Be Defended: Lectures at the Collège de France, 1975-76. New York: Picador.

Foucault, M. (1994) The Order of Things. New York: Vintage.

Foucault, M. (1978) The History of Sexuality, Volume I: An Introduction. New York: Vintage.

Funiciello, T. (1994) Tyranny of Kindness: Dismantling the Welfare System to End Poverty in America. New York: Atlantic Monthly Press.

Garrett, P.M. (2005) Social work’s “electronic turn”: notes on the deployment of information and communication technologies in social work with children and family. Critical Social Policy 25(4): 529-553.

Gilmore, R.W. (2007) Golden Gulag: Prisons, Surplus, Crisis, and Opposition in Globalizing California. Berkeley: University of California Press.

Hacking, I. (2007) Making Up People. London Review of Books 28(16).

Hacking, I. (1990) The Taming of Chance. Cambridge: Cambridge University Press.

Hacking, I. (1982) Biopolitics and the Avalanche of Printed Numbers. Humanities in Society 5(3-4): 279295.

Haggerty, K.D. and R.V. Ericson. (2006) The New Politics of Surveillance and Visibility. Toronto: University of Toronto Press.

Haggerty, K.D. and R.V. Ericson. (2000) The surveillant assemblage. British Journal of Sociology 51(4) : 605-622.

Hardt, M. (1995) The Withering of Civil Society. Social Text 45(14:4):27-43.

INCITE! Women of Color Against Violence. (2007) The Revolution Will Not Be Funded: Beyond the NonProfit Industrial Complex. Boston: South End Press.

Lyon-Callo, V. (2004) Inequality, Poverty, and Neoliberal Governance: Activist Ethnography in the Homeless Sheltering Industry. Toronto: Broadview Press.

Manovich, L. (2001) The Language of New Media. Cambridge, MA: The MIT Press.

Mink, G. (1998) Welfare’s End. Ithaca: Cornell University Press.

Monahan, T. (2006) Surveillance and Security: Technological Politics and power in Everyday Life. New York: Routledge.

Office of the Federal Register. (2004) Department of Housing and Urban Development: Homeless Management Information Systems (HMIS) Data and Technical Standards Final Notice. Federal 
Register 69(146): 45888-45932.

Office of the Federal Register. (2003) Department of Housing and Urban Development: Homeless Management Information Systems (HMIS) Data and Technical Standards Notice. Federal Register 68 (140): 43430-43454.

Parton, N. (2006) Changes in the Form of Knowledge in Social Work: From the 'Social' to the 'Informational'?’ British Journal of Social Work: 1-27.

Piven, F.F. and R. Cloward (1993) Regulating the Poor: The Functions of Public Welfare. New York: Vintage.

Porter, T. (1995) Trust in Numbers: The Pursuit of Objectivity in Science and Public Life. Princeton: Princeton University Press.

Rose, N. (1999) Powers of Freedom: Reframing Political Thought. Cambridge: Cambridge University Press.

Rossi, P.H. (1989) Down and Out in America: The Origins of Homelessness. Chicago: The University of Chicago Press.

Sapey, B. (1997) Social Work Tomorrow: Towards a Critical Understanding of Technology in Social Work. British Journal of Social Work 27: 803-814.

Scott, J.C. (1999) Seeing Like a State: How Certain Schemes to Improve the Human Condition Have Failed. Princeton: Princeton University Press.

Shorter, E. (1997) A History of Psychiatry: From the Era of the Asylum to the Age of Prozac. New York: Wiley \& Sons.

Snow, D.A., L. Anderson and P. Kogel. (1994) Distorting Tendencies in Research on the Homeless. American Behavioral Scientist 37(4): 461-475.

Snow, D.A. .and L. Anderson. (1993) Down on Their Luck: A Study of Homeless Street People. Berkeley: University of California Press.

Strauss, S.E. and F.A. McAlister. (2000) Evidence-based medicine: a commentary on common criticisms. Canadian Medical Association Journal 163(7): 837 - 841.

Tagg, J. (1993) The Burden of Representation. Minneapolis: University of Minnesota Press.

Thacker, E. (2006) The Global Genome: Biotechnology, Politics, and Culture. Cambridge, MA: The MIT Press.

Thacker, E. (2004) Foreword: Protocol is as Protocol Does. In A.R. Galloway, Protocol: How Control Exists After Decentralization. Cambridge, MA: The MIT Press: xxiii-xxii.

Thompson, L.L. (2006) A History of HUD. http://www.mysite.verizon.net/hudhistory.

U.S. Department of Housing and Urban Development. (2007a) The Annual Homeless Assessment Report to Congress.

U.S. Department of Housing and Urban Development. (2007b) Demonstrating the Uses of Homeless Data at the Local Level: Case Studies From Nine Communities (September 2007).

U.S. Department of Housing and Urban Development. (2006a) Fifth Annual Progress Report on HUD's Strategy for Improving Homeless Data Collection, Reporting and Analysis. 
Willse: “Universa I Data Elements"

U.S. Department of Housing and Urban Development. (2006b) Homeless Management Information System (HMIS) Fact Sheet.

Webb, S.A. (2006) Social Work in a Risk Society. London: Palgrave.

Wong, Y.-L. I., J. M. Park, and H. Nemon (2006) Homeless Service Delivery in the Context of Continuum of Care. Administration in Social Work 30(1): 67-94. 